Article

\title{
Bacteria Detection and Differentiation Using Impedance Flow Cytometry
}

\author{
Casper Hyttel Clausen ${ }^{1}$, Maria Dimaki ${ }^{2, *}$, Christian Vinther Bertelsen ${ }^{1,2}$, Gustav Erik Skands ${ }^{1}$, \\ Romen Rodriguez-Trujillo ${ }^{3,4}$, Joachim Dahl Thomsen ${ }^{2}$ and Winnie E. Svendsen ${ }^{2}$ \\ 1 SBT Instruments ApS, Diplomvej 381, 2800 Kgs. Lyngby, Denmark; chc@sbtinstruments.com (C.H.C.); \\ cvb@sbtinstruments.com (C.V.B.); ges@sbtinstruments.com (G.E.S.) \\ 2 Technical University of Denmark, Department of Micro- and Nanotechnology, Oersteds Plads 345 East, \\ DK-2800 Kgs. Lyngby, Denmark; jdth@nanotech.dtu.dk (J.D.T.); wisv@nanotech.dtu.dk (W.E.S.) \\ 3 Department of Electronics and Biomedical Engineering, University of Barcelona. C/Martí i Franquès 1, \\ 08028 Barcelona, Spain; romen.rodriguez@ub.edu \\ 4 Institute for Bioengineering of Catalonia (IBEC), the Barcelona Institute for Science and Technology (BIST), \\ C/Baldiri i Reixac 10-12, 08028 Barcelona, Spain \\ * Correspondence: madi@nanotech.dtu.dk; Tel.: +45-45-256-402
}

Received: 20 September 2018; Accepted: 15 October 2018; Published: 17 October 2018

check for updates

\begin{abstract}
Monitoring of bacteria concentrations is of great importance in drinking water management. Continuous real-time monitoring enables better microbiological control of the water and helps prevent contaminated water from reaching the households. We have developed a microfluidic sensor with the potential to accurately assess bacteria levels in drinking water in real-time. Multi frequency electrical impedance spectroscopy is used to monitor a liquid sample, while it is continuously passed through the sensor. We investigate three aspects of this sensor: First we show that the sensor is able to differentiate Escherichia coli (Gram-negative) bacteria from solid particles (polystyrene beads) based on an electrical response in the high frequency phase and individually enumerate the two samples. Next, we demonstrate the sensor's ability to measure the bacteria concentration by comparing the results to those obtained by the traditional CFU counting method. Last, we show the sensor's potential to distinguish between different bacteria types by detecting different signatures for $S$. aureus and E. coli mixed in the same sample. Our investigations show that the sensor has the potential to be extremely effective at detecting sudden bacterial contaminations found in drinking water, and eventually also identify them.
\end{abstract}

Keywords: electrical impedance spectroscopy; bacteria detection; bacteria differentiation; water quality; bacteria counting

\section{Introduction}

One of the most common ways to determine if bacteria are present in a given sample, e.g., food, urine, blood etc. is to plate the sample on an agar plate and culture it for 1-3 days inside an incubator. This method is extremely easy and precise, but also very time consuming, because you need to wait for the growth to occur before you know the concentration of your sample and can react accordingly.

This problem is of particular importance in drinking water systems [1,2], because the contaminated water can quickly affect a large population. The slow analysis time of a traditional plate count means that the water utilities cannot alert the population that a contamination is present before the potentially dangerous water is consumed. Due to the size of the piping system, it can also be difficult for the utilities to identify the source and location of the contamination and the drinking water can stay polluted for many days. 
Effective enumeration of bacteria in liquid samples without the need for a time consuming culture step has been an issue for several years [3] and a number of methods already exist that address this problem. Some of the most prominent of these are fluorescence flow cytometry [4], electrical impedance spectroscopy [5] and image analysis methods [6]. However, fluorescence flow cytometry requires the use of either stains or labels in order to enumerate bacteria, which complicates the process [7]. Imaging techniques require the development of identification algorithms and are difficult to perform in real time.

Characterizing biological samples by detecting dielectric changes using electrical impedance is a technique that has been used in several forms, including Coulter counters and impedance flow cytometers. Electrical impedance spectroscopy (EIS) [8-11] has been used to investigate different biological samples in suspension and is able to characterize biological sample properties in a label free manner. In EIS, a microfluidic channel is used to direct a sample of particles dispersed in a liquid towards a set of electrodes with an applied AC electrical field [10]. Changes in the electric field during particle transitions depend on the dielectric and structural properties (size, composition) of the particles, which can therefore be determined through interpretation of the measured electrical current.

Different properties of the particles are probed at different frequencies, e.g., the particle size is probed at low frequencies in the $\mathrm{kHz}$ range while the particle composition is probed at higher frequencies in the low $\mathrm{MHz}$ range. Therefore, modern EIS devices apply a mixed multi-frequency signal in order to simultaneously probe the particle properties. Integration of EIS into microsystems is a relatively new development, which has been demonstrated to have various applications within characterization of biological samples [8,9]. The advantages of this integration are better control and higher sensitivity of the system.

The technique has been widely used for analysis of biological material; from differentiation of red blood cells extracted from fish and human leukocytes [12] to detection of DNA in droplets [13]. Gawad et al. [8] presented the first single cell EIS differential microfluidic cell analysis system. They reported the capability of EIS to differentiate erythrocytes and erythrocyte ghost cells, as well as solid particle size separation in continuous flow. Furthermore, the technology has been applied to a broad number of different micro-sized samples; it has been used to distinguish between different yeast cells [14] and human blood cells of different kinds [9,15-17]. EIS has also been used to measure the effect of electrical lysis on yeast cells [18]. Additionally, several reports exist on the modeling of the signal response and how to elucidate how the different properties of the sample influence the recorded signal $[14,19]$. Further applications include differentiating bacteria from polystyrene beads [20] and distinguishing heat treated bacteria samples from un-treated samples [11].

Millions of bacteria species exist, many with unique structural and pathogenic properties. Traditionally, bacteria are divided into two classes, gram negative and gram positive, depending on the ability of their cell wall to retain crystal violet dye. Gram positive bacteria have an outer membrane layer (see Figure 1), which is not present for gram negative bacteria. The presence of the extra membrane is therefore a potential target for differentiation between gram positive and gram negative bacteria by EIS.

In this paper we will present how we can detect and enumerate bacteria in water samples using EIS. We will show that it is possible to detect bacteria not only in artificial buffers (e.g., diluted PBS), but also in tap water. Furthermore, we will show that it is possible to differentiate between gram positive and gram negative bacteria, by using our method to distinguish between two of the most commonly found bacteria. The novelty of the paper lies in the real time continuous detection of bacteria in water samples using impedance, as opposed to publications detecting bacteria in a static environment. 


\section{Materials and Methods}

\subsection{Sample Composition}

The bacteria used in this work are Escherichia coli (E. coli) and Staphylococcus aureus (S. aureus). E. coli is a rod shaped Gram negative bacterium with a length of 2-3 $\mu \mathrm{m} \mathrm{[21]} \mathrm{and} \mathrm{a} \mathrm{diameter} \mathrm{of} 0.5 \mu \mathrm{m}$. It is a common cause for bacterial infections in humans and animals [22]. The cytoplasm of E. coli, which is considered to be electrically conductive, is surrounded by an electrically isolating lipid inner membrane (10 nm [23]), a conductive periplasmic space containing the peptidoglycan wall (20 nm [24]) and another isolating lipid outer membrane (13 nm), see Figure $1 \mathrm{~B}$.

S. aureus is a spherical Gram positive bacterium with a diameter of approximately $1 \mu \mathrm{m}$ [25]. It has a conducting cytoplasm in the center surrounded by a lipid membrane and a peptidoglycan cell wall, characteristic of gram-positive bacteria. The lipid membrane has a thickness of $10 \mathrm{~nm}$ and is normally assumed to be non-conducting, while the cell wall has a thickness of $60 \mathrm{~nm}$ and is assumed to be electrically conducting [24], see Figure 1A.
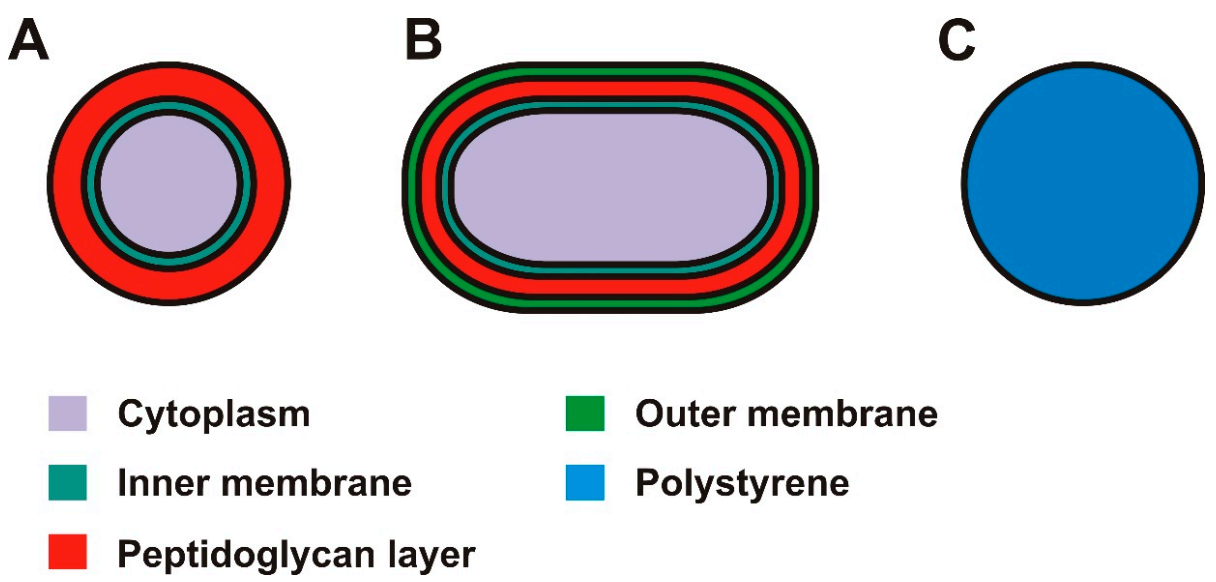

Figure 1. Schematic drawings of the bacteria and particles used in this report (A) Gram-positive S. Aureus (B) Gram- negative E. coli. (C) Polystyrene beads.

The bacteria (Escherichia coli strain INV- $\alpha$ from Invitrogen, Nærum Denmark, methicillin-susceptible Staphylococcus aureus from Statens Serum Institut, Copenhagen, Denmark), were cultured on appropriate pre-prepared agar plates (LB agar, L5542-10EA, Sigma Aldrich Denmark A/S, Copenhagen, Denmark; blood agar, A600, VWR, Copenhagen, Denmark, respectively). The bacteria were spread on the agar plates, dried (10-15 min), and incubated at $37^{\circ} \mathrm{C}$ for $24 \mathrm{~h}$. Before the experiments a small amount of $E$. coli was transferred from the agar plate into individual incubation tubes containing $5 \mathrm{~mL}$ of LB broth and incubated at $37^{\circ} \mathrm{C}$ for $24 \mathrm{~h}$ with rotation $(215 \mathrm{rpm})$. After the $24 \mathrm{~h}$ incubation $1 \mathrm{~mL}$ LB broth containing bacteria was centrifuged, the broth was removed, and the bacteria were re-suspended in either diluted PBS (1/20× PBS, D8537, Sigma Aldrich) or tap water at appropriate concentrations. Polystyrene beads, illustrated in Figure 1C, with diameters of $1 \mu \mathrm{m}$ and $2 \mu \mathrm{m}$ were used as non-biological reference particles. The polystyrene beads are dielectric homogenous spheres which conduct current primarily on their surface [26]. The polystyrene beads were acquired from Polysciences, Inc. (Warrington, PA, USA).

\subsection{Detection Principle}

Figure 2A illustrates the detection principle as well as the electrode configuration on the microfluidic chip. At low frequencies (100-1000 kHz) (Figure 2A, 1 and 2) it is expected that the electrical field will not be able penetrate the beads or the bacteria due to the isolating nature of their bulk and membrane, respectively. The current will only move in the liquid medium and the measured signal will depend on the volume displacement due to the particles or bacteria investigated, 
i.e., the signal depends on particle/bacteria size. At higher frequencies ( $1 \mathrm{MHz}-10 \mathrm{MHz})$, the electric field is still not able to penetrate the bulk of the polystyrene beads (Figure 2A, 3). However, it is capable of partially penetrating the membrane of the bacteria and thus probe the membrane and cytoplasm composition (Figure 2A, 4) [19].

A

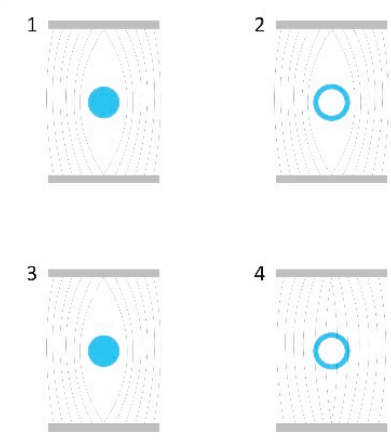

B
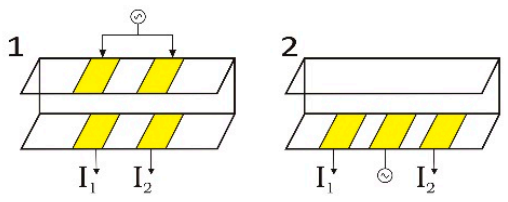

$\mathrm{I}=\mathrm{I}_{1}-\mathrm{I}_{2}$

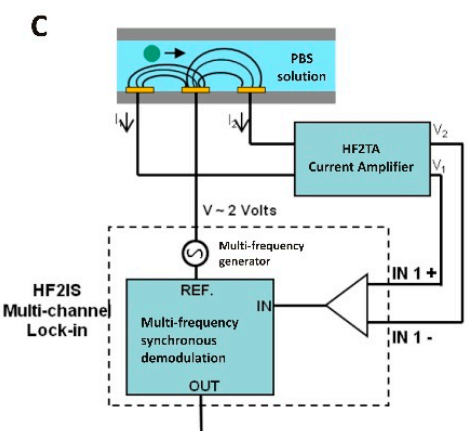

D
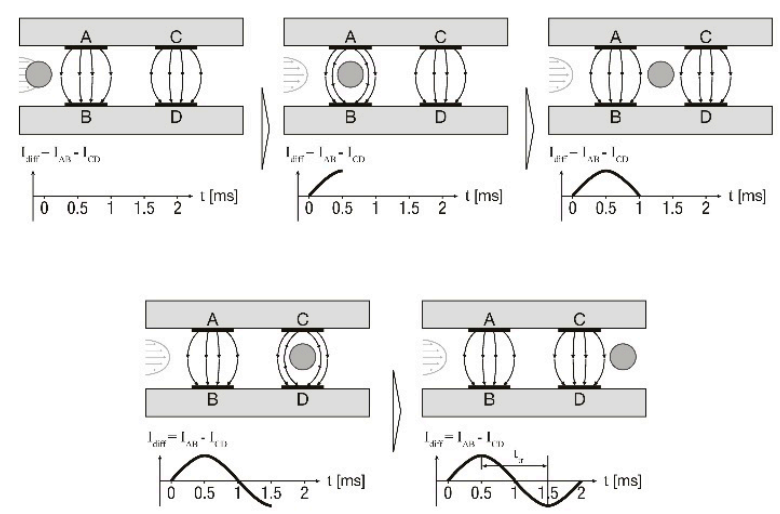

Figure 2. (A) Electric fields generated by two electrodes at low frequencies (1 and 2) and high frequencies ( 3 and 4 ) on beads ( 1 and 3 ) and bacteria (2 and 4). (B) Schematic of the measuring principle using front facing (1) or coplanar (2) electrodes. A differential signal between the two measuring electrodes is recorded for further analysis. (C) Schematic drawing of the measurement setup, in the case of coplanar electrodes. The sample is injected into the system by a syringe pump. A multi-frequency lock-in amplifier is used to generate and detect the signal. The signal from the measuring electrodes is passed through a current preamplifier before it is returned to the lock-in amplifier. (D) Schematic showing the detection principle. When the particle is not influencing the electric field generated by the electrodes, the differential signal is zero. As the particle travels in the channel it will only disturb the field of one set of electrodes at a time, which gives rise to a differential signal. When the particle leaves the electrodes area a transition looking like a sinusoidal signal will have been recorded, indicating the transition of one particle.

\subsection{Chip Fabrication}

Two types of chips were fabricated and used in this work: chips with coplanar electrodes and chips with front facing electrodes (Figure 2B). The fabrication process is similar for the two chip types: Gold electrodes were defined on 4-inch Pyrex wafers by photolithography, e-beam vapor deposition and lift-off, using titanium as an adhesive layer. On top of the electrodes the channels were formed in SU-8 2005 (MicroChem, Berlin, Germany) by negative photolithography as described by Demierre et al. $[27,28]$. The channels were sealed using a second Pyrex wafer (lid wafer) with openings for electrode access and fluidic inlet and outlet defined using powder blasting. The lid wafer used for the front facing electrodes had additionally gold electrodes fabricated as those of the bottom wafer. The lid wafer was then thermally bonded to the bottom wafer as described by Serra et al. [29]. The microchannels were $10 \mu \mathrm{m}$ wide and $10 \mu \mathrm{m}$ high. The front facing electrodes exposed to the 
channel are $10 \mu \mathrm{m}$ long and $10 \mu \mathrm{m}$ wide with a pitch of $16 \mu \mathrm{m}$. The dimensions and pitch are the same for the coplanar electrodes.

\subsection{Measurement Setup}

The setup used in this work uses a layout as shown in Figure 2B. An AC excitation signal is applied to either the top electrodes (Figure 2B 1, front facing) or the middle electrode (Figure 2B 2, coplanar), and the current is measured at the two remaining electrodes. In order to normalize the detected signal a differential measurement is carried out between the two sets of electrodes, giving a measured current of $I=I 1-I 2$, as shown in Figure 2B.

The measurement setup consists of a custom-built aluminum chip holder containing the necessary electrical and fluidic connections (fittings from Upchurch Scientific ${ }^{\circledR}$, IDEX Health and Science, Oak Harbor, WA, USA) for the microfluidic chip. O-rings were used to seal the fluid connections of the chip to the holder. Differential EIS measurements were performed with a HF2IS Impedance Spectroscope (Zurich Instruments, Zurich, Switzerland). The peak differential current during a transition was used as the characterizing parameter of the particle. The signal was amplified by a HF2TA trans-impedance amplifier (Zurich Instruments). A schematic drawing of the setup is shown in Figure 2C. The applied signal was $3 \mathrm{~V}$ (amplitude) with the attenuation of the low pass filter of the lock-in set to $24 \mathrm{~dB}$ with a bandwidth of $502 \mathrm{~Hz}$. The sample rate was $28,800 \mathrm{Sa} / \mathrm{s}$. The preamplifier trans-impedance gain was set to $10 \mathrm{kV} / \mathrm{A}$.

The sample liquid was driven through the chip using a Nexus 3000 syringe pump (Chemyx Inc., Stafford, TX, USA) at a rate of $0.01 \mu \mathrm{L} / \mathrm{min}$. The measurements were carried out at two frequencies simultaneously; a low frequency of $200 \mathrm{kHz}$ and a high frequency of $7 \mathrm{MHz}$. These frequencies were selected based on experimentally recorded spectra at frequencies between $200 \mathrm{kHz}$ and $10 \mathrm{MHz}$ on samples containing different bacteria (E. coli, S. aureus and L. anisa-data shown in Supplementary Figure S1).

\subsection{Samples}

The experiments were generally performed in a low conductivity saline solution (PBS diluted to $1 / 20$ with Milli-Q water) in order to better control the conductivity of the medium and to ensure that the medium was particle free before introducing $E$. coli and polystyrene beads. The conductivity of the prepared saline solution was measured to be $85 \mathrm{mS} / \mathrm{m}$ with a CDM210 conductivity meter (Radiometer Analytical, Lyon, France).

Additionally, experiments in tap water were carried out. The samples were prepared in tap water to demonstrate the capabilities of the biosensor in its envisioned operational environment, considering that solid particles often exist in tap water supply and hence should be distinguishable from bacteria contamination.

The concentration of bacteria (E. coli and/or S. aureus) and beads of 1 and $2 \mu \mathrm{m}$ diameter in the two sample buffers was always $2.5 \times 10^{6} \mathrm{~mL}^{-1}$ for each particle type. The beads were used in order to test the system's ability to discriminate solid particles in the $1 \mu \mathrm{m}$ to $2 \mu \mathrm{m}$ range from bacteria (which have approximately the same size) in tap water.

For the experiments investigating the sample concentration, the bacteria (E. coli) concentration was varied while the concentration of $2 \mu \mathrm{m}$ beads was kept constant at $2 \times 10^{6}$ beads $/ \mathrm{mL}$.

\subsection{Data Acquisition and Analysis}

The raw data was recorded by a computer and analyzed using a custom MATLAB script (MathWorks Inc., Natick, MA, USA), which identifies the differential peak current (Figure 2D) during a particle transition. The program identifies the height and width of the peaks from the transitions for the signal at both frequencies. The concentration of particles $(C)$ is calculated using the transition time ( $\left.t_{\text {trans }}\right)$ (i.e., the time is takes a particle to pass from one measurement electrode to the next) and 
the volume $(V)$ between the electrodes together with the number of events $(N)$ during the time of a measurement $\left(t_{m}\right)$ :

$$
C=\left(N / t_{m}\right) /\left(V / t_{\text {trans }}\right)=\left(N \times t_{\text {trans }}\right) /\left(V \times t_{m}\right)
$$

In this way, the calculated concentration is independent of the flowrate of the sample.

\section{Results and Discussion}

\subsection{Separation of Biological and Non-Biological Samples in Diluted PBS and Tap Water}

Samples in tap water containing E. coli, $1 \mu \mathrm{m}$ and $2 \mu \mathrm{m}$ beads were driven through the chip. Figure 3A,B show a correlation plot of the peak differential current measured at $200 \mathrm{kHz}$ versus the opacity ((high frequency peak differential current)/(low frequency peak differential current)) versus the phase angle response measured at $7 \mathrm{MHz}$, respectively. We observe a clear separation in the measured current response at $200 \mathrm{kHz}$ for $1 \mu \mathrm{m}$ and $2 \mu \mathrm{m}$ beads. E. coli and $1 \mu \mathrm{m}$ beads have roughly the same volume, and thus provide the same current response at the low frequency. As seen in Figure 3A, a plot of the opacity versus current is not able to differentiate between bacteria and beads of the same volume. However, a clear separation between $1 \mu \mathrm{m}$ beads and bacteria can be seen in the phase angle measurements at $7 \mathrm{MHz}$. It is also observed that the $1 \mu \mathrm{m}$ and $2 \mu \mathrm{m}$ beads share the same phase angle response due to their similar bulk composition. It is reasonable to assume that the membrane structure of $E$. coli introduces a phase response at $7 \mathrm{MHz}$, which is different from the signal from the polystyrene beads, which do not have a membrane. This makes the phase angle a potentially useful parameter for differentiating microorganisms from other particles in tap water.
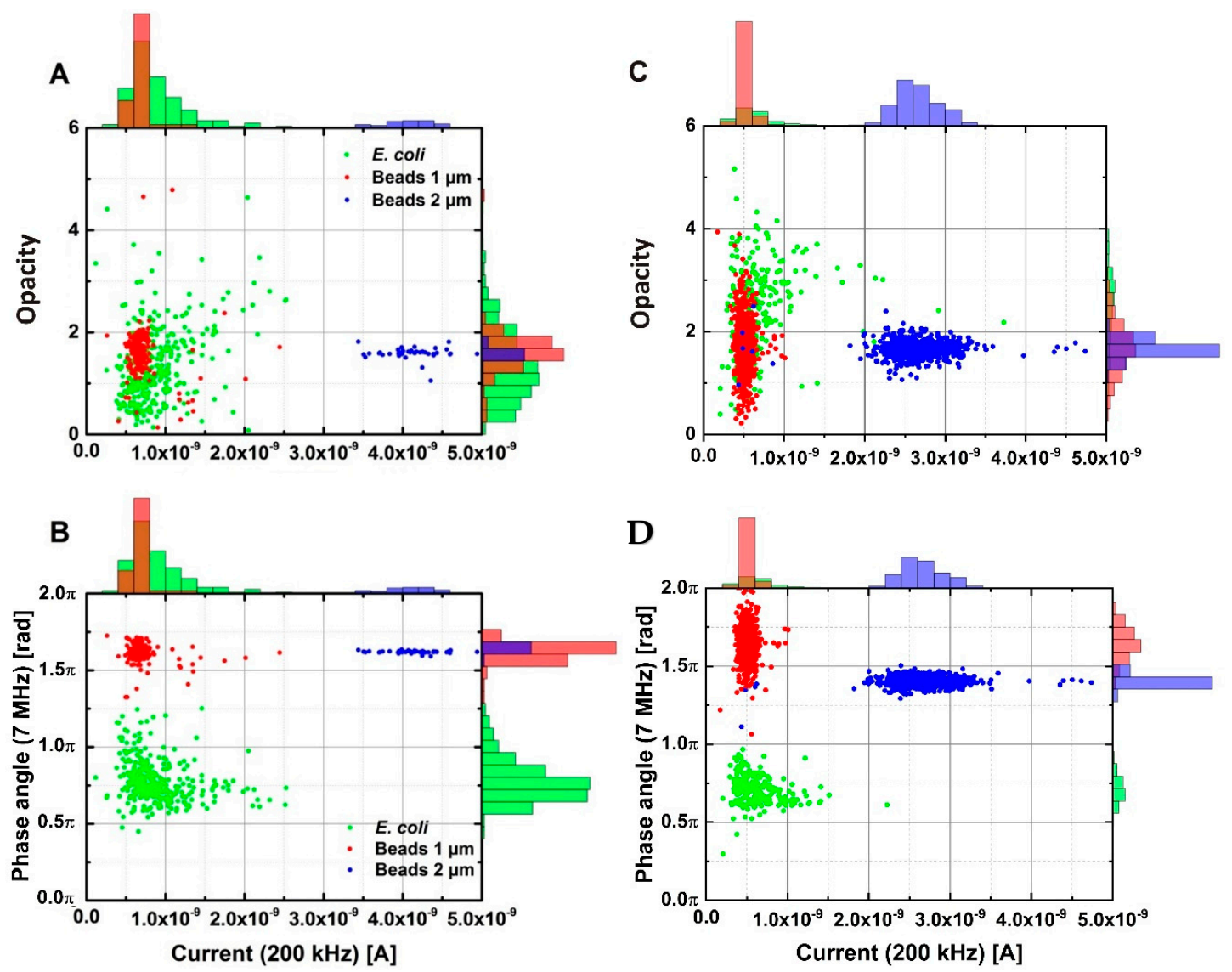

Figure 3. (A) Opacity plotted against the low frequency signal of E. coli and polystyrene beads in drinking water. (B) Phase shift at $7 \mathrm{MHz}$ plotted against the low frequency signal of $E$. coli and polystyrene beads in drinking water. $(\mathbf{C}, \mathbf{D})$ Same as $(\mathbf{A}, \mathbf{B})$, but with the bacteria and beads dispersed in $1 / 20 \times$ PBS. 
Tap water can be a difficult buffer to work with as its composition and electrical properties vary depending on the time of day and the source. In order to have a well-controlled buffer with a conductivity in the same range as tap water we used PBS diluted 20 times with milli-Q water $(1 / 20 \times$ PBS), which has a conductivity of $0.085 \mathrm{~S} / \mathrm{m}$. E. coli, $1 \mu \mathrm{m}$ and $2 \mu \mathrm{m}$ beads were added to the buffer and the impedance response was measured with the sensor. The results are shown in Figure 3C,D. It is evident that the two buffers behave similarly, with the correlation plot of the current at $200 \mathrm{kHz}$ versus the phase angle at $7 \mathrm{MHz}$ still showing a clear separation between bacteria and beads. The small differences in the phase of the 1 and $2 \mu \mathrm{m}$ beads in Figure 3D can most likely be attributed to the function of the filters in the measurement setup and variations in the data analysis algorithm.

Figure 3 confirms that $1 / 20 \times$ PBS is a good substitute to tap water when one wants to control the properties of the liquid better, considering that tap water comes in a variety of conductivities and composition. We note here that the ionic strength (and effectively the conductivity) of the solution has an effect on the recorded high frequency phase. Tap water comes in a range of different conductivities, but it should preferable be between 0 and $150 \mathrm{mS} / \mathrm{m}$ to be suitable for human consumption. In this range of conductivities the phase signal from the bacteria is stable, as is shown in Figure S2 (Supplementary Data), and we can therefore conclude that local variations in drinking water conductivity will not have an effect on the sensor. However, we note that at higher conductivities the phase signal will change, e.g., if PBS is used as the solution, in which case the bacteria can no longer be distinguished from polystyrene beads (Figure S3 in Supplementary Data).

\subsection{Determination of Bacteria Concentration}

It is not essential to know the exact bacteria concentration when detecting sudden contamination events, however, it is very important to be able to detect a change in the bacteria concentration, as it is this change in concentration, which is used to determine if the water supply has been suddenly contaminated. As a result, any bacteria detection system should be capable of rapidly determining any increase in bacteria concentration.

To demonstrate the sensor's ability to accurately measure changes in concentration five samples with varying E. coli concentration and a fixed concentration of $2 \mu \mathrm{m}$ beads were prepared (see Table 1 ). The concentration was then determined with the sensor with a measurement time of 25-30 min per sample. The samples were also plated and the colonies were counted after $24 \mathrm{~h}$. The calculated concentrations by the EIS system (Equation (1)) and the plate count are shown in Table 1.

Table 1. Details of the concentration determination experiments, along with the measured transitions and the calculated concentrations by EIS and plating.

\begin{tabular}{ccccccc}
\hline Sample & Measurement Time (s) & Beads $(\#)$ & Beads $(/ \mathbf{m L})$ & E. coli $(\#)$ & E. coli $(/ \mathbf{m L})$ & Plate Count $(/ \mathbf{m L})$ \\
\hline A & 1551.46 & 377 & 191,202 & 7 & 6221 & 3000 \\
B & 1554.65 & 426 & 201,612 & 92 & 48,181 & 154,000 \\
C & 1551.71 & 398 & 192,603 & 224 & 118,006 & 326,000 \\
D & 1551.9 & 366 & 185,596 & 483 & 274,769 & 643,000 \\
E & 1861.85 & 445 & 186,478 & 843 & 396,767 & $1.00 \times 10^{6}$ \\
\hline
\end{tabular}

The plots shown in Figure 4 depict the measured data of the bacteria concentration as detected by the system and by CFU counting. The bacteria count of the system is not identical to the results obtained with the CFU method, but there is a proportional relation between the measured values by EIS and the values obtained by CFU. This indicates that the difference between the two counting methods is caused by the way the concentration is estimated (see Section 2.6), for example due to variations in the detection volume that arise from fabrication. The result could also be influenced by smaller bacteria, which are missed due to a lack of sensitivity in the system. The $2 \mu \mathrm{m}$ beads are all counted successfully as the signal from these is at least 8 times larger than that for the bacteria. Even though the system is not able to accurately determine the exact concentration of bacteria in a sample, its ability to precisely determine a change in concentration makes it a candidate for monitoring bacteria contaminations 
in liquid samples. Moreover, since the relationship between the actual (by CFU) concentration and measured concentration is linear, then the actual concentration can be accurately estimated after a system calibration.

We note that the discrepancy observed in sample A, where the concentration calculated by EIS is larger than the one found by CFU which arises due to statistical variations in low concentration samples along with the in-built errors in the data processing. Indeed, we note that in the 25 min of the experiment only seven transitions were registered as opposed to about 100 and over in samples B to E. As the data analysis software can erroneously detect up to five transition events in a clean sample, it is likely that the EIS calculated concentration for sample A is overestimated. From Table 1 and using samples B to E we can also estimate our detection limit as the concentration required for detecting a single transition event as $522 \pm 40$ bacteria $/ \mathrm{mL}$.

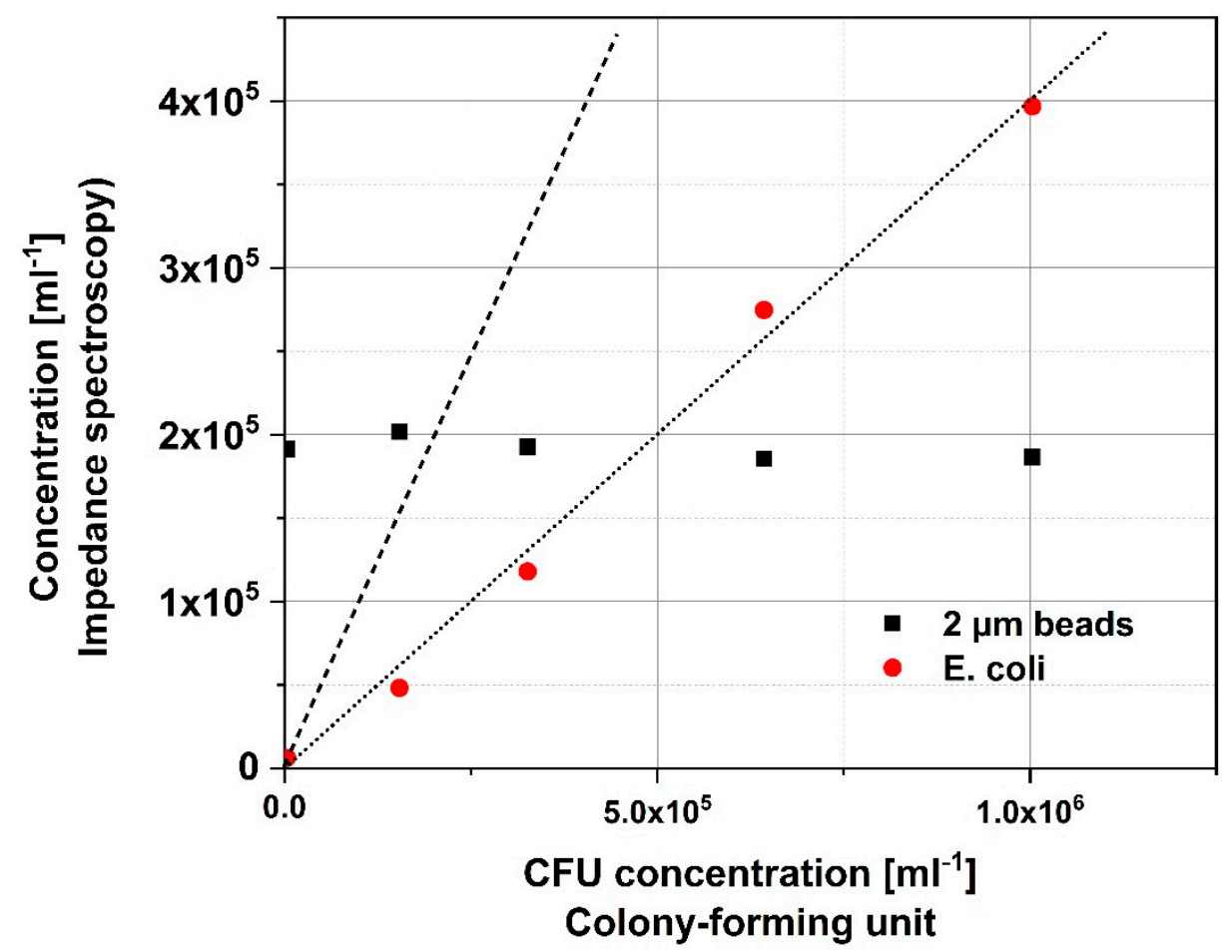

Figure 4. Bacteria concentration measured with the system (impedance spectroscopy) and colony-forming unit counting. The beads were only counted using the system and their concentration was kept constant at $2 \times 10^{5} \mathrm{~mL}^{-1}$. The dashed line represents the 1:1 relation between the two methods. The dotted trend line for the E. coli data has an $R^{2}$ value of 0.9937 .

\subsection{Differentiation between Gram-Positive and Gram-Negative Bacteria}

The structural differences between the membranes of Gram-positive and Gram-negative bacteria make it probable that a difference will appear on the high frequency phase signal. This is indeed the case, as can be shown in Figure 5. Figure 5A shows the correlation plot for three samples, one containing E. coli mixed with polystyrene beads, one containing S. aureus (MSSA) and one containing a mixed population of E. coli, MSSA and beads. In Figure 5A we can see that distinct populations appear around 2, 3 and $5 \mathrm{rad}$, with the population at 5 rad visible in all the samples, suggesting that this population comes from the polystyrene beads that were present in two of the samples. As the MSSA sample did not contain beads, it is plausible that there is some bead contamination in the system, giving rise to the bead signal in the MSSA sample.

By plotting the number of points appearing at the different phase angles in Figure 5B, we can see that E. coli and MSSA indeed present two distinct populations at 2 and 3 rad, respectively. Fitting the data using normal distributions we find that the mean phase value for the E. coli is 2.22 rad with a 
standard deviation of $0.55 \mathrm{rad}(\mathrm{n}=325)$, while the mean phase value for MSSA is 3.09 rad with a standard deviation of $0.26 \mathrm{rad}(\mathrm{n}=241)$. The two means are statistically significantly different as the $p$-value is of the order of $10^{-90}$. However, we note that the tails for the two distributions overlap at approximately the mean $+1 \times$ standard deviation for $E$. coli and the mean $-1 \times$ standard deviation for MSSA. Assuming a Gaussian distribution for the two populations, this means that we can correctly identify and categorize $84 \%$ of the bacteria in the sample.

Whether or not the differentiation method can be generalized to a larger number of different bacteria is still under investigation. Theoretically, different bacteria types have different membrane and cytoplasm structures and should therefore have different dielectric properties, though admittedly the differences are small. However, increasing the system sensitivity can be achieved by changing the channel geometry (e.g., in [30]), using a different set of frequencies or combining EIS with other dielectric based methods, such as DEP.

\subsection{General Discussion}

In the above we have presented how our device can detect and differentiate E. coli and S. aureus in water samples, however, the limit of detection (LOD) is far from what is required for such a sensor. The acceptable limit is 1 coliform per $100 \mathrm{~mL}$ water which is much lower than the ca. 500 bacteria $/ \mathrm{mL}$ that our device can measure. Although E. coli and S. aureus were used as test bacteria in order to validate our method against the standard plate count, the purpose of this device is not to reach this type of sensitivity for a single bacteria species but to provide the total bacteria count in a water sample and detect changes to this number. Indeed, there are four main indicators used for water quality [31]: (a) Heterotrophic plate count (HPC), which refers to the number of culturable bacteria in a water sample, (b) Total coliform, (c) Fecal coliform and (d) E. coli. The presented sensor aims to address the first indicator, HPC [32]. Significant changes in HPC serve as an alert for possible deterioration of water quality, triggering further investigation. However, HPC only measures culturable bacteria, with the total number of bacteria being 1000 to 10,000 times higher [33]. The presented device can measure all bacteria in water, culturable or not, and therefore provides a fast and accurate measurement of the total bacteria count, that does not involve time-consuming culturing steps. The Danish limits for HPC are 5 bacteria per $\mathrm{mL}$ at $37^{\circ} \mathrm{C}$, which means a total of 5000 to 50,000 bacteria per $\mathrm{mL}$, which is easily achievable with the presented device.

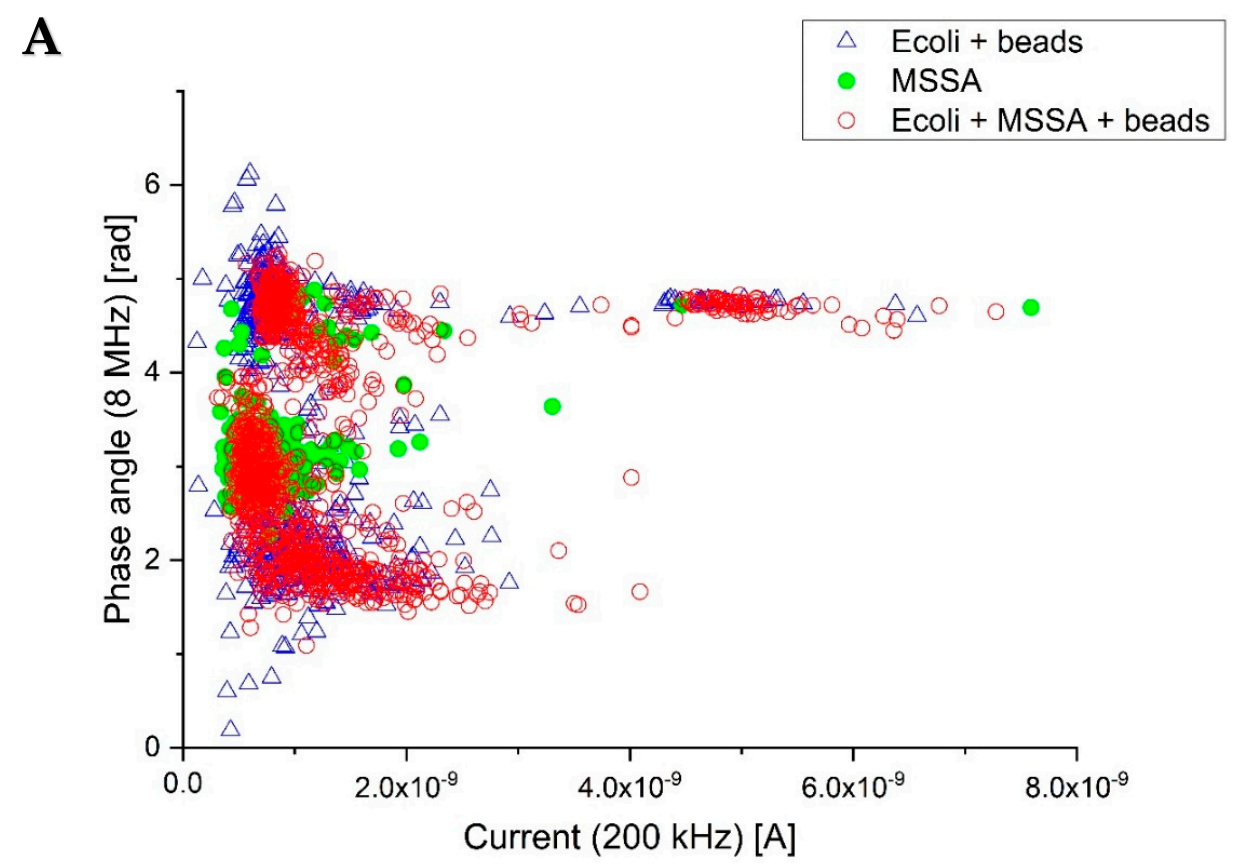

Figure 5. Cont. 


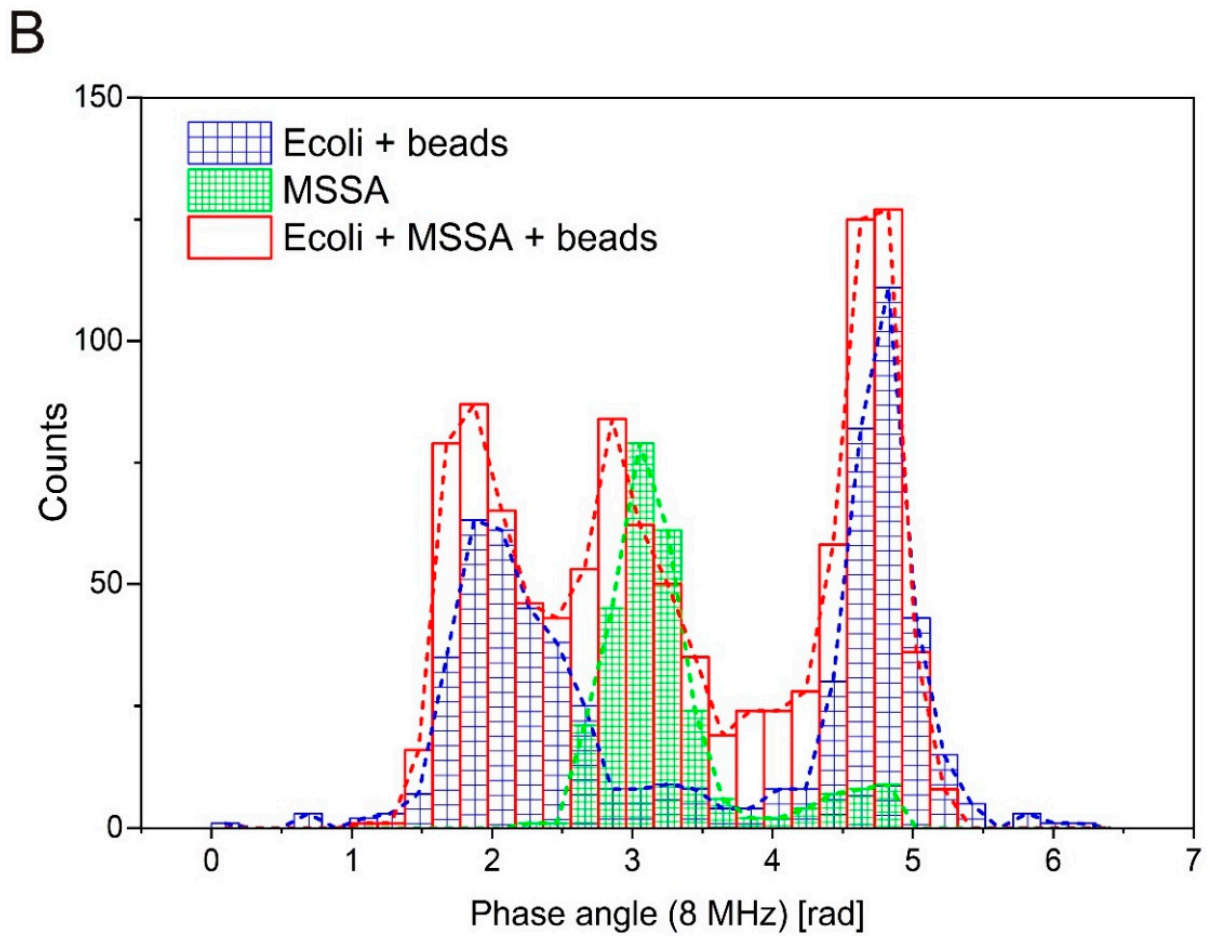

Figure 5. (A) Phase shift at $8 \mathrm{MHz}$ plotted against the low frequency signal of the bacteria E. coli and S. aureus and polystyrene beads in diluted PBS (B) Histogram of the high frequency phase angle, clearly showing the two populations of bacteria.

The maximum continuous and flawless operation time for this device installed in a water pipe was 14 days, which is not adequate for a real life application. Future work will focus on improving the stability of the system and on establishing the functionality in real-world samples.

\section{Conclusions}

In conclusion, a microfluidic chip was fabricated and used to distinguish E. coli from solid particles represented by polystyrene beads in both tap water as well as a diluted saline solution using multi-frequency impedance spectroscopy. Using the phase angle response at a frequency of 7 or $8 \mathrm{MHz}$, E. coli bacteria could be separated from polystyrene beads on a single transition basis. Moreover, we were able to distinguish between gram-positive and gram-negative bacteria using this method. Together with the system's ability to detect bacteria concentrations with an LOD of $522 \mathrm{~mL}^{-1}$ and its ability to accurately determine a change in bacteria concentration, the system has been demonstrated to be an interesting candidate for further operational testing. All measurements were obtained with the label free technique of electrical impedance flow cytometry, and the results show great promise for using electrical impedance flow cytometry as the detection principle of the next generation of online and real-time water quality control sensors.

Supplementary Materials: The following are available online at http:/ /www.mdpi.com/1424-8220/18/10/3496/s1.

Author Contributions: Conceptualization, C.V.B., G.E.S., R.R.-T. and J.D.T.; Data curation, C.H.C., M.D., C.V.B., G.E.S. and J.D.T.; Formal analysis, M.D. and C.V.B.; Funding acquisition, W.E.S.; Investigation, C.H.C., C.V.B. and G.E.S.; Project administration, W.E.S.; Supervision, R.R.-T. and W.E.S.; Validation, C.V.B.; Writing-original draft, C.H.C. and M.D.; Writing-review \& editing, M.D., C.V.B., J.D.T. and W.E.S.

Funding: The research was supported by the Carlsberg Foundation (grant no. 2012_01_0191) and the Novo Nordisk foundation (grant no. 9659).

Conflicts of Interest: The authors declare no conflict of interest. The funders had no role in the design of the study; in the collection, analyses, or interpretation of data; in the writing of the manuscript, or in the decision to publish the results. 


\section{References}

1. Højris, B.; Christensen, S.C.; Albrechtsen, H.J.; Smith, C.; Dahlqvist, M. A novel, optical, on-line bacteria sensor for monitoring drinking water quality. Sci. Rep. 2016, 6, 23935. [CrossRef] [PubMed]

2. Lopez-Roldan, R.; Tusell, P.; Cortina, J.L.; Courtois, S. On-line bacteriological detection in water. TrAC Trends Anal. Chem. 2013, 44, 46-57. [CrossRef]

3. Hargesheimer, E.E.; Conio, O.; Popovicova, J.; Proaqua, C. Online Monitoring for Drinking Water Utilities; American Water Works Association: Denver, CO, USA, 2002.

4. Hammes, F.; Egli, T. Cytometric methods for measuring bacteria in water: Advantages, pitfalls and applications. Anal. Bioanal. Chem. 2010, 397, 1083-1095. [CrossRef] [PubMed]

5. Gawad, S.; Cheung, K.; Seger, U.; Bertsch, A.; Renaud, P. Dielectric spectroscopy in a micromachined flow cytometer: Theoretical and practical considerations. Lab Chip 2004, 4, 241-251. [CrossRef] [PubMed]

6. Olesen, T.; Valvik, M.C.; Larsen, N.A.; Sandberg, R.H.; Unisensor, A.S. Optical Sectioning of a Sample and Detection of Particles in a Sample. U.S. Patent 8,780,181, 15 July 2014.

7. Díaz, M.; Herrero, M.; García, L.A.; Quirós, C. Application of flow cytometry to industrial microbial bioprocesses. Biochem. Eng. J. 2010, 48, 385-407. [CrossRef]

8. Gawad, S.; Schild, L.; Renaud, P.H. Micromachined impedance spectroscopy flow cytometer for cell analysis and particle sizing. Lab Chip 2001, 1, 76-82. [CrossRef] [PubMed]

9. Holmes, D.; Pettigrew, D.; Reccius, C.H.; Gwyer, J.D.; van Berkel, C.; Holloway, J.; Davies, D.E.; Morgan, H. Leukocyte analysis and differentiation using high speed microfluidic single cell impedance cytometry. Lab Chip 2009, 9, 2881-2889. [CrossRef] [PubMed]

10. Sun, T.; Morgan, H. Single-cell microfluidic impedance cytometry: A review. Microfluid. Nanofluid. 2010, 8, 423-443. [CrossRef]

11. David, F.; Hebeisen, M.; Schade, G.; Franco-Lara, E.; Di Berardino, M. Viability and membrane potential analysis of Bacillus megaterium cells by impedance flow cytometry. Biotechnol. Bioeng. 2012, 109, 483-492. [CrossRef] [PubMed]

12. Ayliffe, H.E.; Frazier, A.B.; Rabbitt, R.D. Electric impedance spectroscopy using microchannels with integrated metal electrodes. J. Microelectromec. Syst. 1999, 8, 50-57. [CrossRef]

13. Lin, R.; Simon, M.G.; Lee, A.P.; Lopez-Prieto, J. Label-free detection of DNA amplification in droplets using electrical impedance. In Proceedings of the 15th International Conference on Miniaturized Systems for Chemistry and Life Sciences, Seattle, WA, USA, 2-6 October 2011; Volume 3, pp. 1683-1685.

14. Haandbæk, N.; Bürgel, S.C.; Heer, F.; Hierlemann, A. Characterization of subcellular morphology of single yeast cells using high frequency microfluidic impedance cytometer. Lab Chip 2014, 14, 369-377. [CrossRef] [PubMed]

15. Holmes, D.; Morgan, H. Single Cell Impedance Cytometry for Identification and Counting of CD4 T-Cells in Human Blood Using Impedance Labels. Anal. Chem. 2010, 82, 1455-1461. [CrossRef] [PubMed]

16. Evander, M.; Ricco, A.J.; Morser, J.; Kovacs, G.T.; Leung, L.L.; Giovangrandi, L. Microfluidic impedance cytometer for platelet analysis. Lab Chip 2013, 13, 722-729. [CrossRef] [PubMed]

17. Kirkegaard, J.; Clausen, C.H.; Rodriguez-Trujillo, R.; Svendsen, W.E. Study of Paclitaxel-Treated HeLa Cells by Differential Electrical Impedance Flow Cytometry. Biosensors 2014, 4, 257-272. [CrossRef] [PubMed]

18. Mernier, G.; Hasenkamp, W.; Piacentini, N.; Renaud, P. Multiple-frequency impedance measurements in continuous flow for automated evaluation of yeast cell lysis. Sens. Actuators B Chem. 2012, 170, $2-6$. [CrossRef]

19. Morgan, H.; Sun, T.; Holmes, D.; Gawad, S.; Green, N.G. Single cell dielectric spectroscopy. J. Phys. D Appl. Phys. 2007, 40, 61-70. [CrossRef]

20. Haandbæk, N.; Bürgel, S.C.; Heer, F.; Hierlemann, A. Resonance-enhanced microfluidic impedance cytometer for detection of single bacteria. Lab Chip 2014, 14, 3313-3324. [CrossRef] [PubMed]

21. Grossman, N.; Ron, E.Z.; Woldringh, C.L. Changes in cell dimensions during amino acid starvation of Escherichia coli. J. Bacteriol. 1982, 152, 35-41. [PubMed]

22. Freeman, J.T.; Anderson, D.J.; Sexton, D.J. Seasonal peaks in Escherichia coli infections: Possible explanations and implications. Clin. Microbiol. Infect. 2009, 15, 951-953. [CrossRef] [PubMed] 
23. Mitra, K.; Ubarretxena-Belandia, I.; Taguchi, T.; Warren, G.; Engelman, D.M. Modulation of the bilayer thickness of exocytic pathway membranes by membrane proteins rather than cholesterol. Proc. Natl. Acad. Sci. USA 2004, 101, 4083-4088. [CrossRef] [PubMed]

24. Sanchis, A.; Brown, A.P.; Sancho, M.; Martinez, G.; Sebastian, J.L.; Munoz, S.; Miranda, J.M. Dielectric characterization of bacterial cells using dielectrophoresis. Bioelectromagnetics 2007, 28, 393-401. [CrossRef] [PubMed]

25. Wyatt, P.J. Cell Wall Thickness, Size Distribution, Refractive Index Ratio and Dry Weight Content of Living Bacteria (Stapylococcus aureus). Nature 1970, 226, 277-279. [CrossRef] [PubMed]

26. Park, S.; Zhang, Y.; Wang, T.H.; Yang, S. Continuous dielectrophoretic bacterial separation and concentration from physiological media of high conductivity. Lab Chip 2011, 11, 2893-2900. [CrossRef] [PubMed]

27. Demierre, N.; Braschler, T.; Linderholm, P.; Seger, U.; van Lintel, H.; Renaud, P. Characterization and optimization of liquid electrodes for lateral dielectrophoresis. Lab Chip 2007, 7, 355-365. [CrossRef] [PubMed]

28. Moresco, J.; Clausen, C.H.; Svendsen, W. Improved anti-stiction coating of SU-8 molds. Sens. Actuators B Chem. 2010, 145, 698-701. [CrossRef]

29. Serra, S.; Schneider, A.; Malecki, K. A simple bonding process of SU-8 to glass to seal a microfuidic device. In Proceedings of the Multi-Material Micro Manufacture, Borovets, Bulgaria, 3-5 October 2007; pp. $43-46$.

30. Clausen, C.H.; Skands, G.E.; Bertelsen, C.V.; Svendsen, W.E. Coplanar Electrode Layout Optimized for Increased Sensitivity for Electrical Impedance Spectroscopy. Micromachines 2015, 6, 110-120. [CrossRef]

31. Verhille, S. Understanding Microbial Indicators for Drinking Water Assessment: Interpretation of Test Results and Public Health Significance. National Collaborating Center for Environmental Health. Available online: http:/ / www.ncceh.ca/sites/default/files/Microbial_Indicators_Jan_2013_0.pdf (accessed on 16 October 2018).

32. World Health Organisation (WHO). Guidelines for Drinking-Water Quality Incorporating First Addendum, 3rd ed.; WHO: Geneva, Switzerland, 2006; Volume 1, p. 285.

33. Staley, J.T. Measurement of in situ activities of nonphotosynthetic microorganisms in aquatic and terrestrial habitats. Ann. Rev. Microbiol. 1985, 39, 321-346. [CrossRef] [PubMed] 